\title{
Fünf Jahre nach neuer Weiterbildungsordnung (WBO 2005): Erfahrungen aus dem Seminar Basischirurgie, Common Trunk in Bielefeld
}

\author{
Five Years after the New Further Education \\ Legislation (WBO 2005): Experience from the \\ Seminar Basic Surgery, Common Trunk in Bielefeld
}

\section{Einleitung \\ $\nabla$}

In diesem Jahr bereits zum fünften Mal hat das Evangelische Krankenhaus Bielefeld im Auftrag des Berufsverbandes der Deutschen Chirurgen und mit Unterstützung der Vereinigung NiederrheinischWestfälischer Chirurgen einen Facharztkurs für Basischirurgie (sog. CommonTrunk-Kurs) im Jugendgästehaus und Bildungszentrum Dürkopp Tor6 in Bielefeld organisiert.

Das Ziel der kurzen kompakten Übersicht ist es, im Sinne einer „Nachlese“ Werden, Wachsen, initiale Erkenntnisse und abgeleitete bzw. umgesetzte Konsequenzen im Konzept des vor 5 Jahren inaugurierten Common-Trunk-Kurses darzustellen. Dieser Kurs ist einer der heutigen Eckpfeiler und Positiverfahrungen der chirurgischen Fort- und (Facharzt-)Weiterbildung neben Anderen [1-8] durch Vermittlung der Vororterfahrungen aus Bielefeld. Er fand auch statt vor dem zusätzlichen Hintergrund, weiterführende Empfehlungen für eine kontinuierliche Optimierung des Weiterbildungskurses, nicht zuletzt als Denkanstoß auch für die übrigen, vom BDC veranstalteten Common-Trunk-Kurse, zu geben.

\section{Evidenzbasis}

$\nabla$

Als Basis für diesen Kurs diente anfangs die erste, ab 2007 die zweite Auflage des Buches „Facharztprüfung Chirurgie: in Fragen, Fällen und Antworten“, herausgegeben von Reymond, Lippert und Haas [9]. In den letzten Jahren hat dieses Evidenzbasierte Werk eine breite Akzeptanz im deutschsprachigen Europa gefunden und wird bei den Facharztprüfungen als Referenzopus vermehrt angewendet. Es wurde also sukzessive versucht, die Synergieeffekte zu nutzen, die zwischen dem Buch und dem Facharztkurs entstehen. So werden die Vorträge jährlich nach neuer Stu- dienlage aktualisiert, was wiederum die laufende Vorbereitung der 3. Auflage des Buches erleichtert. Nach Absolvierung des Kurses können die Teilnehmer das Buch als Begleiter für ihre chirurgische Weiterbildung nutzen, um die Erinnerung an die Vorträge immer wieder zu aktivieren.

\section{Inhalte und Pädagogik $\nabla$}

Der einwöchige Common-Trunk-Kurs, der jährlich im März organisiert wird, besteht aus 36 vollen Arbeitsstunden, davon 16 Stunden Theorie, 17 Stunden Workshops und 3 Stunden Evaluation. Der Inhalt des Kurses wurde nach der neuen Weiterbildungsordnung (WBO 2005 [10]) gestaltet. So sind 53\% der Lerneinheiten der perioperativen Medizin gewidmet, $22 \%$ der Allgemein- und Viszeralchirurgie, $13 \%$ der Unfallchirurgie, $7 \%$ der Gefäßchirurgie und $5 \%$ der Thoraxchirurgie. Es mussten im Jahr 2006 richtungsweisende Entscheidungen getroffen werden, weil die WBO zwar die Themen, aber nicht die genauen Inhalte vorschreibt. Insbesondere war die Trennlinie zwischen Common Trunk (die ersten 2 Jahre der Weiterbildung) und der sogenannten Allgemeinen Chirurgie (Jahr 3 bis 6) nicht immer einfach zu ziehen. Derselben inhaltlichen Unschärfe sind wir allerdings auch bei der Herausgabe der 2. Auflage des „Facharztbuches Chirurgie“ begegnet. Der Kurs wurde nach Vorgaben der Fortbildungsakademie des Berufsverbandes der Deutschen Chirurgen und der Deutschen Gesellschaft für Chirurgie - soweit damals vorhanden - gestaltet. Es muss jedoch erwähnt werden, dass auch innerhalb dieser Gremien keine Einigkeit bestand hinsichtlich der Inhalte, der lernpädagogischen Methoden (Projektorientiertes Lernen vs. Lehrunterricht, physischer Unterricht vs. e-learning, usw.) oder der Form (einwöchiger vs. modularer Kurs).

\section{Referenten}

$\nabla$

Als Referenten für diesen Kurs wurden möglichst Kapitelautoren des Facharztbuches, aber auch Chef- und Oberärzte des gastgebenden und berichtenden Klinikums, der Westfälischen Krankenhäuser und aus dem Valeo-Verbund (Verbund Evangelischer Krankenhäuser in Westfalen) eingeladen. Insgesamt haben jährlich 50 bis 60 Referenten und Tutoren die etwa 70Teilnehmer begleitet. Traditionell wird der Kurs durch einen Motivationsvortrag eines Lehrstuhlinhabers eröffnet, mit z.T. provokanten Titeln wie z.B. bei dem Vortrag von Prof. N.Senninger in 2007: „Ich denke, deswegen bin ich... Chirurg“. Täglich werden die Beurteilungen durch die Teilnehmer gesammelt und ausgewertet, die Vortragenden erhalten nach Kursende ihr Ergebnis. Jeder Referent erhält sein Ranking (in einem geschlossenen Briefumschlag) mit einem persönlichen Brief. Unsere Erfahrung zeigt, dass die Referenten mit hohem Interesse auf die Benotung gewartet haben: Es ist nicht die Regel, dass ca.100\% der Zuhörer ein Feedback geben. Diese Rückmeldung ist offensichtlich auch für den Referenten von großer Bedeutung. Um die Qualität der Vorträge stets zu verbessern, haben wir uns dazu entschieden, die drei letzten Referenten im darauf folgenden Jahr nicht mehr einzuladen, dies öffnet die Tür für neue Chefärzte. Allerdings muss hier erwähnt werden, dass manche Themen wie Medizintechnologie, OP-Lagerung oder DRG traditionell schlecht abgeschnitten haben, dies lag am Thema und nicht an den Vortragenden. Als Kursorganisatoren haben sich Prof. Reymond und Priv.-Doz. Steinert dazu entschieden, diese Themen selbst zu übernehmen, um die Kollegen nicht zu benachteiligen.

\section{Stärkung des Weiterbildungs- standortes \\ $\nabla$}

Da die Benotung des Kurses, der Workshops und der Betreuung in den letzten 5 Jahren stets zu den bestnotierten der BDC-Kurse gehörte, hat dadurch der Ausbildungsstandort Westfalen einen anerkannteren Stellenwert erlangt. Dieses gemeinsame Angebot eines CommonTrunk-Kurses durch chirurgische Mitbewerber wird inzwischen auch von den teilnehmenden Chefärzten stark unterstützt, die Einladung als Referent ist zum Qualitätsmerkmal geworden. Auch wenn der Kurs sicherlich die Möglichkeit bietet, 
sich bei den jungen Kollegen positiv darzustellen, haben wir uns untersagt, die Veranstaltung als Job-Börse zu missbrauchen. So werden die Referenten motiviert, das Layout ihrer Folien mit dem BDC-Logo zu gestalten und auf jegliche „Werbebotschaften“ zu verzichten: Die Referenten sind im Kurs, um jüngere Kollegen auszubilden und nicht, um sich selbst zu profilieren oder um Mitarbeiter zu gewinnen.

\section{Teilnehmer \\ $\nabla$}

In den letzten 5 Jahren haben insgesamt ca. 350 Teilnehmerinnen und Teilnehmer aus der gesamten Bundesrepublik das Seminar Basischirurgie in Bielefeld besucht und von einem vielfältigen theoretischen und praktischen Programm profitiert. Besonders geschätzt wurde das individuelle Feedback zur eigenen Leistung: Die theoretischen Kenntnisse werden täglich getestet und die individuellen Fertigkeiten am Nachmittag ebenfalls begutachtet. Mit einer Ausnahme ist es jedes Jahr gelungen, einen Laparoskopie-Simulator im Rahmen des MIC-Workshops einzusetzen, an dem jeder Teilnehmer seine handwerkliche Geschicklichkeit messen lassen konnte. Der Einsatz moderner Phantome beim notfallmedizinischen Workshop hat ebenfalls eine Rückmeldung über den Erfolg der Reanimationsmaßnahmen erlaubt. Für die besten Leistungen in der praktischen Arbeit wurden am Ende des Kurses 3 Preise vergeben.

\section{Methodologie}

$\nabla$

Dieser Kurs wurde nach bewährten pädagogischen Prinzipien gestaltet, wie sie zum Beispiel im traditionsreichen gastroenterologischen Kurs in Davos seit Jahrzehnten verfeinert wurden. Die theoretischen Inhalte werden knapp und präzise durch Kurzvorträge im Frontalunterricht vermittelt. Diese neuen Kenntnisse werden am nächsten Vormittag durch ein Quiz zu den am Vortage gehörten Themen reflektiert, um so eine bessere Einprägung zu erreichen und die erworbenen Kenntnisse zu testen. Außerdem werden die Teilnehmer so motiviert, rechtzeitig zu erscheinen. Am Ende des Kurses wurden die ersten 3 Quizsieger mit einem attraktiven Preis belohnt, um somit die Lernmotivation der Teilnehmerinnen und Teilnehmer zu erhöhen. Unmittelbar nach dem Quiz wurde eine schriftliche, detaillierte

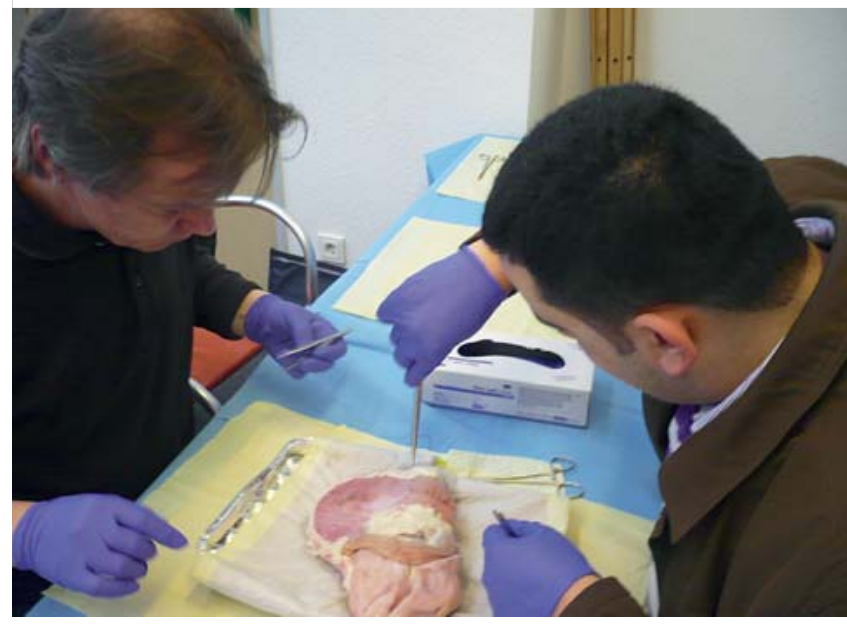

Abb. 1 Realitätsnaher Nahtkurs am Schweindarm.

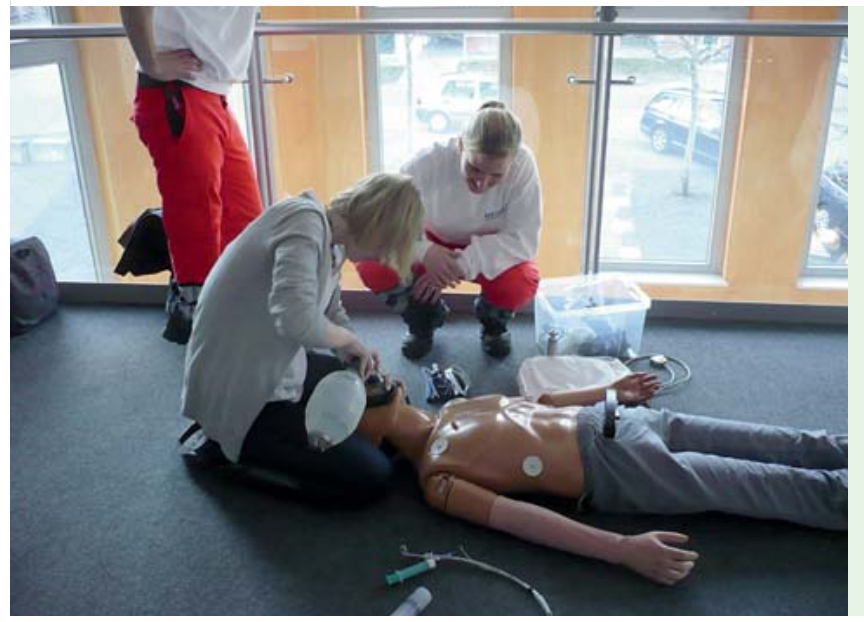

Abb. 2 Szene aus kardipulmonaler Reanimationsübung und Sicherung von Vitalfunktionen.

Bewertung der Vorträge und Workshops des vorigen Tages durch die Teilnehmer vorgenommen.

Nach Kursende wurden die Vorträge, veranlasst durch den BDC, auf eine CD gebrannt, die die Teilnehmer gegen einen geringen Kostenbeitrag erwerben können, um interessante Themen nachzuarbeiten.

\section{Workshops \\ $\nabla$}

Praktische Übungen in den nachmittags stattfindenden Workshops lockern die Atmosphäre auf und erlauben es, nicht nur die theoretischen Kenntnisse, sondern auch die eigenen chirurgischen Fertigkeiten zu testen und zu verbessern. Es werden jeweils 4 Workshops mit ausreichend Arbeitsplätzen für alle Teilnehmer angeboten.

Nahtmaterial, Nahtkurs, Elektrochirurgie, Klammernahttechniken Hier werden die praktischen Aspekte der Naht- und Klammerkunde sowie der HFChirurgie erläutert und geübt. Die Teil- nehmer kommen in Kontakt mit verschiedenen Dissektionstechniken und können am Gewebe selbst deren Wirkung testen. In diesem Workshop können die Teilnehmerinnen an richtigen Organen (Darm, Leber) üben, was immer wieder auf eine positive Resonanz stößt.

\section{Lebensrettende Maßnahmen, Schockraum}

Dieser Workshop wird von Notärzten, von der Berufsfeuerwehr und von professionellen Rettungssanitätern betreut. Geleitet wird der Workshop von Herrn Dr. med. Korth, Träger des Bundesverdienstkreuzes, der für die Ausbildung von Notärzten, Rettungssanitätern und Feuerwehrleuten im Großraum Bielefeld verantwortlich ist. Er war auch bei Massenveranstaltungen wie dem Weltjugendtag oder der Love-Parade in Duisburg im Einsatz. In diesem Workshop werden die wichtigsten Prinzipien der ATLS vermittelt. Danach kann jeder Teilnehmer unter fachkundiger Leitung die Reanimationstechniken, inkl. der Intubationstechnik, vier Stunden lang praktizieren. Im Anschluss wird die Bergung von Schwerver- 
letzten geübt. Ein Notarztwagen und ein RTW stehen zur Verfügung, um einen realitätsnahen Einsatz zu trainieren. Verständlicherweise bekommt dieser Workshop bessere Noten bei schönem Wetter als bei Regen oder Kälte, die im März in Bielefeld noch bissig sein kann.

Verbandlehre, Gipskurs, Enterostroma, VAC-Verbände, Wundversorgung

In einem speziell ausgestatteten Raum werden die verschiedenen Verband- und Gipstechniken demonstriert und von jedem Teilnehmer angewendet. Praktische Anweisungen zum Thema „Gestaltung und Versorgung eines Enterostomas“ werden vermittelt, das Anlegen von VakuumVerbänden wird demonstriert und geübt. Vor 2 Jahren ist ein Arbeitsplatz zum Thema Wundversorgung hinzugekommen, an dem über die Versorgung verschiedener Wunden (z.B. Dekubitus, Ulcus cruris) anhand des TIME-Leitfadens Tissue (Gewebe), Infection (Infektion), Moisture (Exsudatmanagement) und Edge (Förderung Re-Epithelisierung) zur Versorgung chronischer Wunden informiert und an Gemüse geübt wird.

\section{Minimalinvasive Chirurgie}

Hier werden Basisübungen in der MIC vorgeschlagen, um die operativen Fertigkeiten jedes Teilnehmers zu verbessern. Eine ausreichende Anzahl an Arbeitsplätzen (Pelvi-Trainern) ist gesichert, sodass jeder Teilnehmer praktische Übungen durchführen kann z.B. mit Paprikaschoten, Gummibärchen, Streichhölzern usw. Zuerst werden Übungen unter direkter Sicht (dreidimensionale Vision) vorgeschlagen und dann unter Video-Kontrolle (2-dimensionale Sicht) ausgeführt. Die bereits geübten oder besonders geschickten Teilnehmer können auch die laparoskopische Knotentechnik üben. Wie oben erwähnt, wird der Workshop durch eine individuelle Leistungsmessung am Simulator ergänzt.

\section{Einsatz der Industrie}

$\nabla$

Die verschiedenen Workshops werden halbtageweise durch Unternehmen, die im chirurgischen Bereich aktiv sind und Interesse an der Weiterbildung der zukünftigen Chirurgen haben, übernommen. Die Industrie zeigt Interesse, frühzeitig Kontakt mit den angehenden Chirurgen zu knüpfen und die eigenen Produkte vorzustellen. Nur mit dem materiel- len und personellen Einsatz der Industrie sind die Organisatoren in der Lage, die Teilnahmegebühren gering zu halten. So kommen z.B. Klammernahtgeräte in ausreichender Menge für alle Teilnehmer zum Einsatz. Auch wurde angestrebt, die Einweisung nach Medizinproduktegesetz vorzunehmen, die Teilnehmer erhalten dafür vom Veranstalter einen Gerätepass. Allerdings erklären sich nicht alle Mitarbeiter der Industrie dazu bereit, die Teilnehmer in der verfügbaren Zeit einzuweisen. Die Organisatoren haben die Vertreter der Industrie darauf aufmerksam gemacht, dass die Veranstaltung eine Weiterbildungs- und keine Werbeveranstaltung ist. In der Regel wurde dieses Prinzip beachtet, allerdings gab es hier und da kritische Bemerkungen von Teilnehmern, die eine sofortige Korrektur zur Folge hatten. Grundsätzlich hat sich aber die Zusammenarbeit mit der Industrie harmonisch gestaltet unter strikter Einhaltung der gesetzlichen Vorschriften und des Ehrenkodexes. Die Sponsorengelder wurden ausschließlich durch den Berufsverband in Berlin und nicht durch den Veranstalter akquiriert.

\section{Veranstaltungsort}

$\nabla$

Das Seminar findet in dem sehr schönen Jugendgästehaus und Bildungszentrum Bielefeld statt, welches anlässlich der Expo 2000 umgebaut wurde. Das helle und freundliche, zentral gelegene Gästehaus zeichnet sich durch eine anspruchsvolle Architektur aus Glas und Stahl aus. Verbunden durch eine gläserne Brücke mit dem hochmodernen Aus- und Weiterbildungszentrum liegt es im denkmalgeschützten Teil einer ehemaligen Fahrradfabrik. Wichtiger als die Räumlichkeiten selbst ist jedoch die Möglichkeit, direkt am Tagungsort auch übernachten zu können. Das Jugendgästehaus bietet 2und 3-Bettzimmer mit einem 2-SterneHotelstandard zu einem unschlagbaren VP-Preis, z.B. Übernachtung im DZ und Vollpension für 43,30€. Aufgrund des gemeinsamen Lebens während des einwöchigen Kurses hat sich in jedem Jahr eine positive Stimmung und Lernkultur entwickelt.

\section{Rahmenprogramm $\nabla$}

Ziel des Rahmenprogrammes ist es, ein gegenseitiges Kennenlernen und einen Austausch zwischen den Teilnehmerin- nen und Teilnehmern und auch mit den Tutoren zu ermöglichen. Nachdem den ganzen Tag konzentriert gearbeitet wurde, gibt es am Abend Erholung pur. Ein Teilnehmer sagte: Die Stimmung ist wie in einem olympischen Dorf. Die Mitbewerber werden Gleichgesinnte. Es besteht für alle hinreichend die Möglichkeit, sich über die positiven und negativen Erfahrungen im eigenen Krankenhaus auszutauschen. Auch neue Freundschaften werden geschlossen, insbesondere nach der inzwischen legendären Donnerstagsparty: Ein klasse Orchester sorgt dafür, dass auf der Diele getanzt wird. Es wird sogar gemunkelt, dass der Common-Trunk-Kurs in Bielefeld tatsächlich für Paarbildung und - wer weiß - für chirurgischen Nachwuchs gesorgt haben könnte.

\section{Weitere Entwicklungen \\ $\nabla$}

Inzwischen werden zahlreiche Weiterbildungskurse für chirurgische Berufsanfänger angeboten, sei es vom Berufsverband Deutscher Chirurgen, von der Deutschen Gesellschaft für Chirurgie oder von anderen Anbietern. Dieses breite Angebot ist sicher prinzipiell zu begrüßen, wie man weiß, belebt Konkurrenz das Geschäft. Allerdings möchten die Organisatoren des Common-Trunk-Kurses in Bielefeld vorsichtig darauf hinweisen, dass ein zu reiches Angebot in eine Verschwendung von Ressourcen und Zeit münden könnte, was nicht wünschenswert ist. Es gibt - leider - nur eine begrenze Anzahl an jungen Medizinern, die sich für den chirurgischen Beruf entscheiden. Dem Nachwuchs soll Qualität - und nicht nur Quantität - angeboten werden. Es ist also die Aufgabe unserer Führungsgremien, die Weiterbildungslandschaft so zu organisieren, dass der wertvolle, ehrenamtliche Einsatz der Organisatoren, Referenten und Tutoren im Dienst unseres Nachwuchses gewürdigt wird. Das weitere Multiplizieren des Kursangebotes für Berufsanfänger könnte sonst zu halbleeren Seminarsälen führen und schlussendlich zu qualitativen Einbußen.

\section{Interessenkonflikt: Nein}

D. Lemmer ${ }^{1}$, R. Steinert ${ }^{1}$, F. Meyer ${ }^{2}$, M. Beshay ${ }^{1}$, M. A. Reymond ${ }^{1}$

${ }^{1}$ Evangelisches Krankenhaus Bielefeld, Klinik für Allgemein-, Viszeral- und Thoraxchirurgie, Bielefeld, Deutschland

2 Universitätsklinikum Magdeburg A. ö.R., Klinik für Allgemein-, Viszeral- \& Gefäßchirurgie, Magdeburg, Deutschland. 


\section{Literatur}

1 Bärthel E, Schöne U, Scheuerlein H. Wie sollte die chirurgische Ausbildung idealerweise konzipiert sein? - Antworten und Anregungen aus Sicht des Assistenten. Zentralbl Chir 2010; 135: 464-466

2 Brauer RB, Harnoss J-C, Lang J et al. Qualität und Qualitätssicherung der Lehre in der Chirurgie - Empfehlungen aus einem Workshop der Chirurgischen Arbeitsgemeinschaft für Qualitätssicherung. Zentralbl Chir 2010; 135: 18-24

3 Brücher BLDM. Theodor-Billroth-Akademie ${ }^{\circledR}$ Aktive Nachwuchsförderung zukünftiger Chirurgen. Zentralbl Chir 2010; 135: 458-463

4 Niedermeyer $M$. Der Chirurg als Trainer und Mentor. Zentralbl Chir 2010; 135: 475-479

5 Pape-Koehler C, Chmelik C, Åslund AM et al. OP-Lehre multimedial und interaktiv: Webop - ein Ansatz zur Verbesserung der chirur- gischen Weiterbildung. Zentralbl Chir 2010; 135: 467-471

6 Pape-Köhler C, Chmelik C, Rose M et al. Moderne Didaktik in der chirurgischen Weiterbildung - zwischen Anspruch und Wirklichkeit. Zentralbl Chir 2010; 135: 575-579

7 Scheuerlein H, Settmacher U. Gedanken zur Aus- und Weiterbildung zum Chirurgen gestern, heute und morgen. Zentralbl Chir 2010; 135: 451-457

8 Schlein U, Hager-Van Der Laan J. Kommunikation ist der kritische Erfolgsfaktor (nicht nur) in der Aus- und Weiterbildung. Zentralbl Chir 2010; 135: 472-474

9 Reymond MA, Lippert H, Haas N, Hrsg. Facharztprüfung Chirurgie: in Fällen, Fragen und Antworten.; 2. Aufl. München: Elsevier; 2007

10 Weiterbildungsordnung 2005. www.aekwl. de (12.07.2011)
Bibliografie

DOI http://dx.doi.org/10.1055/s-0031-1283715

Online-Publikation: 23.02.2013

Zentralbl Chir 2013; 138: 1-4

(c) Georg Thieme Verlag KG Stuttgart · New York . ISSN 0044-409X

\section{Korrespondenzadresse}

Prof. Dr. med. Marc A. Reymond

Evangelisches Krankenhaus Bielefeld .

Klinik für Allgemein- und Viszeralchirurgie Schildescher Str. 99

33611 Bielefeld

Deutschland

Tel.: $+49 /(0) 521 / 77277401$

Fax: $+49 /(0) 521 / 77277402$

marc.reymond@yahooevkb.de 